\title{
Inequalities for mappings whose second derivativesare quasi-convex or $h$-convex functions
}

M. E. Özdemir, Mustafa Gürbüz, and Çetin Yildiz 


\title{
INEQUALITIES FOR MAPPINGS WHOSE SECOND DERIVATIVES ARE QUASI-CONVEX OR $h$-CONVEX FUNCTIONS
}

\author{
M. EMIN ÖZDEMIR, MUSTAFA GÜRBÜZ, AND ÇETIN YILDIZ
}

Received 30 November, 2012

\begin{abstract}
In this paper, we establish some new integral inequalities for quasi-convex and $h$-convex functions by using a kernel and fairly elementary analysis.

2010 Mathematics Subject Classification: 26A51; 26D15

Keywords: Hermite-Hadamard inequality, quasi-convex functions, $h$-convex functions, weighted Hölder's inequality
\end{abstract}

\section{INTRODUCTION}

One of the most famous inequality for convex functions is so called HermiteHadamard's inequality as follows: Let $f: I \subseteq \mathbb{R} \rightarrow \mathbb{R}$

be a convex function defined on the interval $I$ of real numbers and $a, b \in I$, with $a<b$. Then

$$
f\left(\frac{a+b}{2}\right) \leq \frac{1}{b-a} \int_{a}^{b} f(x) d x \leq \frac{f(a)+f(b)}{2} .
$$

For recent results, refinements, counterparts, generalizations and new Hermite-Hadamard type inequalities see [9], [2],[4] and [11].

Definition 1 (See [7]). A function $f:[a, b] \rightarrow \mathbb{R}$ is said quasi-convex on $[a, b]$ if

$$
f(\lambda x+(1-\lambda) y) \leq \sup \{f(x), f(y)\}, \quad(Q C)
$$

holds for all $x, y \in[a, b]$ and $\lambda \in[0,1]$.

Definition 2 (see [10]). Let $h: J \subseteq \mathbb{R} \rightarrow \mathbb{R}$ be a non-negative function. We say that $f: I \subseteq \mathbb{R} \rightarrow \mathbb{R}$ is an $h$-convex function or that $f$ belongs to the class $S X(h, I)$, if $f$ is nonnegative and for all $x, y \in I$ and $\alpha \in[0,1]$ we have

$$
f(\alpha x+(1-\alpha) y) \leq h(\alpha) f(x)+h(1-\alpha) f(y) .
$$

In recent years, several authors have been studied on integral inequalities. One of the well known of these inequalities is Simpson's inequality as following: 
Theorem 1. Let $f:[a, b] \rightarrow \mathbb{R}$ be a four times continuously differentiable mapping on $(a, b)$ and $\left\|f^{(4)}\right\|_{\infty}=\sup _{x \in(a, b)}\left|f^{(4)}(x)\right|<\infty$. Then, the following inequality holds:

$$
\left|\frac{1}{3}\left[\frac{f(a)+f(b)}{2}+2 f\left(\frac{a+b}{2}\right)\right]-\frac{1}{b-a} \int_{a}^{b} f(x) d x\right| \leq \frac{1}{2880}\left\|f^{(4)}\right\|_{\infty}(b-a)^{4} .
$$

For recent refinements, counterparts, generalizations and new Simpson's type inequalities, see the papers [8], [1], [3], [5].

In [6], Özdemir et al. proved the following lemma:

Lemma 1. Let $f: I \subset \mathbb{R} \rightarrow \mathbb{R}$ be a twice differentiable mapping on $I^{\circ}$ such that $f^{\prime \prime} \in L_{1}[a, b]$, where $a, b \in I$ with $a<b$ and $r \in \mathbb{R}^{+}$then the following equality holds:

$$
\begin{aligned}
& \frac{1}{r(r+1)}[f(a)+f(b)]+\frac{2}{r+1} f\left(\frac{a+b}{2}\right)-\frac{2}{r(b-a)} \int_{a}^{b} f(x) d x \\
& =(b-a)^{2} \int_{0}^{1} k(t) f^{\prime \prime}(t b+(1-t) a) d t
\end{aligned}
$$

where

$$
k(t)=\left\{\begin{array}{lc}
\frac{t}{r}\left(\frac{1}{r+1}-t\right) & , \quad t \in\left[0, \frac{1}{2}\right) \\
(1-t)\left(\frac{t}{r}-\frac{1}{r+1}\right), & t \in\left[\frac{1}{2}, 1\right]
\end{array} .\right.
$$

In this article, by using functions whose second derivatives' absolute values are quasi-convex or $h$-convex, we obtained new inequalities related to the left hand side of Simpson inequality. Also new inequalities are proved.

\section{RESUlts FOR QUASI-CONVEX Functions}

Theorem 2. Let $f: I \subset \mathbb{R} \rightarrow \mathbb{R}$ be a twice differentiable mapping on $I^{\circ}$ such that $f^{\prime \prime} \in L_{1}[a, b]$ and $\left|f^{\prime \prime}\right|^{q}$ is quasi-convex, where $a, b \in I$ with $a<b, r \geq 1$ and $q \geq 1$, then one has the following inequality;

$$
\begin{aligned}
& \left|\frac{1}{r(r+1)}[f(a)+f(b)]+\frac{2}{r+1} f\left(\frac{a+b}{2}\right)-\frac{2}{r(b-a)} \int_{a}^{b} f(x) d x\right| \\
& \leq \frac{r^{3}-3 r+6}{12 r(r+1)^{3}}(b-a)^{2}\left(\sup \left\{\left|f^{\prime \prime}(a)\right|^{q},\left|f^{\prime \prime}(b)\right|^{q}\right\}\right)^{\frac{1}{q}} .
\end{aligned}
$$


Proof. Suppose that $q=1$, from Lemma 1 and by using quasi-convexity of $\left|f^{\prime \prime}\right|$, we obtain

$$
\begin{aligned}
& \left|\frac{1}{r(r+1)}[f(a)+f(b)]+\frac{2}{r+1} f\left(\frac{a+b}{2}\right)-\frac{2}{r} \int_{0}^{1} f(t b+(1-t) a) d t\right| \\
& \leq(b-a)^{2}\left\{\int_{0}^{\frac{1}{2}}\left|\frac{t}{r}\left(\frac{1}{r+1}-t\right)\right|\left|f^{\prime \prime}(t b+(1-t) a)\right| d t\right. \\
& \left.+\int_{\frac{1}{2}}^{1}\left|(1-t)\left(\frac{t}{r}-\frac{1}{r+1}\right)\right|\left|f^{\prime \prime}(t b+(1-t) a)\right| d t\right\} \\
& \leq(b-a)^{2}\left\{\int_{0}^{\frac{1}{2}}\left|\frac{t}{r}\left(\frac{1}{r+1}-t\right)\right| \sup \left\{\left|f^{\prime \prime}(a)\right|,\left|f^{\prime \prime}(b)\right|\right\} d t\right. \\
& \left.+\int_{\frac{1}{2}}^{1}\left|(1-t)\left(\frac{t}{r}-\frac{1}{r+1}\right)\right| \sup \left\{\left|f^{\prime \prime}(a)\right|,\left|f^{\prime \prime}(b)\right|\right\} d t\right\} \\
& =\frac{r^{3}-3 r+6}{12 r(r+1)^{3}}(b-a)^{2} \sup \left\{\left|f^{\prime \prime}(a)\right|,\left|f^{\prime \prime}(b)\right|\right\} .
\end{aligned}
$$

Now suppose that $q>1$, from Lemma 1 we have

$$
\begin{aligned}
& \left|\frac{1}{r(r+1)}[f(a)+f(b)]+\frac{2}{r+1} f\left(\frac{a+b}{2}\right)-\frac{2}{r} \int_{0}^{1} f(t b+(1-t) a) d t\right| \\
& \leq(b-a)^{2} \int_{0}^{1}|k(t)|\left|f^{\prime \prime}(t b+(1-t) a)\right| d t
\end{aligned}
$$

By using Power mean inequality we get

$$
\begin{aligned}
& \left|\frac{1}{r(r+1)}[f(a)+f(b)]+\frac{2}{r+1} f\left(\frac{a+b}{2}\right)-\frac{2}{r} \int_{0}^{1} f(t b+(1-t) a) d t\right| \\
& \leq(b-a)^{2}\left(\int_{0}^{1}|k(t)| d t\right)^{1-\frac{1}{q}}\left(\int_{0}^{1}|k(t)|\left|f^{\prime \prime}(t b+(1-t) a)\right|^{q} d t\right)^{\frac{1}{q}} .
\end{aligned}
$$


Since $\left|f^{\prime \prime}\right|^{q}$ is quasi-convex, we have

$$
\begin{aligned}
& \left|\frac{1}{r(r+1)}[f(a)+f(b)]+\frac{2}{r+1} f\left(\frac{a+b}{2}\right)-\frac{2}{r} \int_{0}^{1} f(t b+(1-t) a) d t\right| \\
& \leq \frac{r^{3}-3 r+6}{12 r(r+1)^{3}}(b-a)^{2}\left(\sup \left\{\left|f^{\prime \prime}(a)\right|^{q},\left|f^{\prime \prime}(b)\right|^{q}\right\}\right)^{\frac{1}{q}} .
\end{aligned}
$$

This completes the proof.

Corollary 1. Under the assumptions of Theorem 2, if we choose $r=1$ in (2.1), we have the following inequality:

$$
\begin{aligned}
& \left|\frac{f(a)+f(b)}{2}+f\left(\frac{a+b}{2}\right)-\frac{2}{b-a} \int_{a}^{b} f(x) d x\right| \\
& \leq \frac{(b-a)^{2}}{24}\left(\sup \left\{\left|f^{\prime \prime}(a)\right|^{q},\left|f^{\prime \prime}(b)\right|^{q}\right\}\right)^{\frac{1}{q}} .
\end{aligned}
$$

Corollary 2. If we take $q=1$ and $r=1$ in (2.1), we obtain an inequality which includes the left-hand side of the Corollary 3 in [6]:

$$
\begin{aligned}
& \left|\frac{f(a)+f(b)}{2}+f\left(\frac{a+b}{2}\right)-\frac{2}{b-a} \int_{a}^{b} f(x) d x\right| \\
& \leq \frac{(b-a)^{2}}{24} \sup \left\{\left|f^{\prime \prime}(a)\right|,\left|f^{\prime \prime}(b)\right|\right\} \\
& \leq \frac{(b-a)^{2}}{24}\left(\left|f^{\prime \prime}(a)\right|+\left|f^{\prime \prime}(b)\right|\right)
\end{aligned}
$$

Corollary 3. If we choose $r=2$ in (2.1), we obtain

$$
\begin{aligned}
& \left|\frac{1}{6}\left[f(a)+4 f\left(\frac{a+b}{2}\right)+f(b)\right]-\frac{1}{b-a} \int_{a}^{b} f(x) d x\right| \\
& \leq \frac{(b-a)^{2}}{81}\left(\sup \left\{\left|f^{\prime \prime}(a)\right|^{q},\left|f^{\prime \prime}(b)\right|^{q}\right\}\right)^{\frac{1}{q}} \\
& \leq \frac{(b-a)^{2}}{81}\left(\left|f^{\prime \prime}(a)\right|^{q}+\left|f^{\prime \prime}(b)\right|^{q}\right)^{\frac{1}{q}}
\end{aligned}
$$

Let $a_{1}=\left|f^{\prime \prime}(a)\right|^{q}, b_{1}=\left|f^{\prime \prime}(b)\right|^{q}$. Here $0<\frac{1}{q}<1$ for $q>1$, using the fact that $\sum_{k=1}^{n}\left(a_{k}+b_{k}\right)^{s} \leq \sum_{k=1}^{n}\left(a_{k}\right)^{s}+\sum_{k=1}^{n}\left(b_{k}\right)^{s}$ for $0 \leq s \leq 1, a_{1}, a_{2}, \ldots, a_{n} \geq 0$, 
$b_{1}, b_{2}, \ldots, b_{n} \geq 0$, we get

$$
\begin{aligned}
& \left|\frac{1}{6}\left[f(a)+4 f\left(\frac{a+b}{2}\right)+f(b)\right]-\frac{1}{b-a} \int_{a}^{b} f(x) d x\right| \\
& \leq \frac{(b-a)^{2}}{81}\left(\left|f^{\prime \prime}(a)\right|+\left|f^{\prime \prime}(b)\right|\right) .
\end{aligned}
$$

Theorem 3. Let $f: I \subset \mathbb{R} \rightarrow \mathbb{R}$ be a twice differentiable mapping on $I^{\circ}$ such that $f^{\prime \prime} \in L_{1}[a, b]$. If $\left|f^{\prime \prime}\right|^{p}$ is quasi-convex on $[a, b]$, where $a, b \in I$ with $a<b, r \geq 1$ and $p>1$, then the following inequality hold

$$
\begin{aligned}
& \left|\frac{1}{r(r+1)}[f(a)+f(b)]+\frac{2}{r+1} f\left(\frac{a+b}{2}\right)-\frac{2}{r(b-a)} \int_{a}^{b} f(x) d x\right| \\
& \leq \frac{(b-a)^{2}}{4}\left(\sup \left\{\left|f^{\prime \prime}(a)\right|^{p},\left|f^{\prime \prime}(b)\right|^{p}\right\}\right)^{\frac{1}{p}} \\
& \times\left(\frac{1}{2}\right)^{\frac{1}{p}}\left(\frac{1}{r(r+1)^{\frac{q+2}{q}}}\right)\left\{\frac{(r-1)^{q+1}\left[2(q+2)-(r-1)(q+1)+2^{2+q}\right]}{(q+1)(q+2)}\right\}^{\frac{1}{q}}
\end{aligned}
$$

where $\frac{1}{p}+\frac{1}{q}=1$.

Proof. By using Lemma 1 and triangle inequality, we have

$$
\begin{aligned}
& \left|\frac{1}{r(r+1)}[f(a)+f(b)]+\frac{2}{r+1} f(r a c a+b 2)-\frac{2}{r(b-a)} \int_{a}^{b} f(x) d x\right| \\
& \leq(b-a)^{2}\left\{\left|\int_{0}^{\frac{1}{2}} \frac{t}{r}\left(\frac{1}{r+1}-t\right) f^{\prime \prime}(t b+(1-t) a) d t\right|\right. \\
& \left.+\left|\int_{\frac{1}{2}}^{1}(1-t)\left(\frac{t}{r}-\frac{1}{r+1}\right) f^{\prime \prime}(t b+(1-t) a) d t\right|\right\} \\
& =(b-a)^{2}\left\{\left|\int_{0}^{\frac{1}{2}} f^{\prime \prime}(t b+(1-t) a)\left(\frac{1}{r+1}-t\right) \frac{t}{r} d t\right|\right.
\end{aligned}
$$




$$
\left.+\left|\int_{\frac{1}{2}}^{1} f^{\prime \prime}(t b+(1-t) a)\left(\frac{t}{r}-\frac{1}{r+1}\right)(1-t) d t\right|\right\} \text {. }
$$

By using weighted version of Hölder's inequality which is described as

$$
\left|\int_{I} f(s) g(s) h(s) d s\right| \leq\left(\int_{I}|f(s)|^{p} h(s) d s\right)^{\frac{1}{p}}\left(\int_{I}|g(s)|^{q} h(s) d s\right)^{\frac{1}{q}}
$$

for $p>1, p^{-1}+q^{-1}=1, h$ is non-negative on $I$ and provided all the other integrals exist and are finite; we have

$$
\begin{aligned}
& \left|\frac{1}{r(r+1)}[f(a)+f(b)]+\frac{2}{r+1} f\left(\frac{a+b}{2}\right)-\frac{2}{r(b-a)} \int_{a}^{b} f(x) d x\right| \\
& \leq(b-a)^{2} \\
& \times\left\{\left(\int_{0}^{\frac{1}{2}}\left|f^{\prime \prime}(t b+(1-t) a)\right|^{p} \frac{t}{r} d t\right)^{\frac{1}{p}}\left(\int_{0}^{\frac{1}{2}}\left|\frac{1}{r+1}-t\right|^{q} \frac{t}{r} d t\right)^{\frac{1}{q}}\right. \\
& \left.+\left(\int_{\frac{1}{2}}^{1}\left|f^{\prime \prime}(t b+(1-t) a)\right|^{p}(1-t) d t\right)^{\frac{1}{p}}\left(\int_{\frac{1}{2}}^{1}\left|\frac{t}{r}-\frac{1}{r+1}\right|^{q}(1-t) d t\right)^{\frac{1}{q}}\right\} .
\end{aligned}
$$

By using the quasi-convexity of $\left|f^{\prime \prime}\right|^{p}$, we get

$$
\begin{aligned}
& \left|\frac{1}{r(r+1)}[f(a)+f(b)]+\frac{2}{r+1} f\left(\frac{a+b}{2}\right)-\frac{2}{r(b-a)} \int_{a}^{b} f(x) d x\right| \\
& \leq(b-a)^{2}\left(\sup \left\{\left|f^{\prime \prime}(a)\right|^{p},\left|f^{\prime \prime}(b)\right|^{p}\right\}\right)^{\frac{1}{p}} \\
& \times\left\{\left(\int_{0}^{\frac{1}{2}} \frac{t}{r} d t\right)^{\frac{1}{p}}\left(\int_{0}^{\frac{1}{2}}\left|\frac{1}{r+1}-t\right|^{q} \frac{t}{r} d t\right)^{\frac{1}{q}}\right.
\end{aligned}
$$




$$
\begin{aligned}
& \left.+\left(\int_{\frac{1}{2}}^{1}(1-t) d t\right)^{\frac{1}{p}}\left(\int_{\frac{1}{2}}^{1}\left|\frac{t}{r}-\frac{1}{r+1}\right|^{q}(1-t) d t\right)^{\frac{1}{q}}\right\} \\
& =\frac{(b-a)^{2}}{4}\left(\sup \left\{\left|f^{\prime \prime}(a)\right|^{p},\left|f^{\prime \prime}(b)\right|^{p}\right\}\right)^{\frac{1}{p}} \\
& \times\left(\frac{1}{2}\right)^{\frac{1}{p}}\left(\frac{1}{r(r+1)^{\frac{q+2}{q}}}\right)\left\{\frac{(r-1)^{q+1}\left[2(q+2)-(r-1)(q+1)+2^{2+q}\right]}{(q+1)(q+2)}\right\}^{\frac{1}{q}} .
\end{aligned}
$$

So the proof is complete.

Corollary 4. Under the assumptions of Theorem 3, if we choose $r=1$ in the inequality (2.2), we have the following inequalities:

$$
\begin{aligned}
& \left|\frac{f(a)+f(b)}{2}+f\left(\frac{a+b}{2}\right)-\frac{2}{b-a} \int_{a}^{b} f(x) d x\right| \\
& \leq \frac{(b-a)^{2}}{16}\left(\frac{1}{2}\right)^{\frac{1}{q}}\left(\sup \left\{\left|f^{\prime \prime}(a)\right|^{p},\left|f^{\prime \prime}(b)\right|^{p}\right\}\right)^{\frac{1}{p}} \\
& \leq \frac{(b-a)^{2}}{16}\left(\sup \left\{\left|f^{\prime \prime}(a)\right|^{p},\left|f^{\prime \prime}(b)\right|^{p}\right\}\right)^{\frac{1}{p}}
\end{aligned}
$$

where $\left(\frac{1}{2}\right)^{\frac{1}{q}} \leq 1, q \in(1, \infty)$.

Corollary 5. If we choose $r=2$ in (2.2), we obtain

$$
\begin{aligned}
& \left|\frac{1}{6}\left[f(a)+4 f\left(\frac{a+b}{2}\right)+f(b)\right]-\frac{1}{b-a} \int_{a}^{b} f(x) d x\right| \\
& \leq \frac{(b-a)^{2}}{24}\left(\frac{1}{2}\right)^{\frac{1}{p}}\left(\frac{1}{3}\right)^{\frac{2}{q}}\left(\frac{(q+3)+2^{q+2}}{(q+1)(q+2)}\right)\left(\sup \left\{\left|f^{\prime \prime}(a)\right|^{p},\left|f^{\prime \prime}(b)\right|^{p}\right\}\right)^{\frac{1}{p}} .
\end{aligned}
$$

3. RESUltS FOR $h$-CONVEX FUnCTIONS

Theorem 4. Let $f: I \subset \mathbb{R} \rightarrow \mathbb{R}$ be a twice differentiable mapping on $I^{\circ}$ such that $f^{\prime \prime} \in L_{1}[a, b]$ and $\left|f^{\prime \prime}\right|$ is $h$-convex, where $a, b \in I$ with $a<b$ and $r \geq 1$, then one has the following inequality;

$$
\left|\frac{1}{r(r+1)}[f(a)+f(b)]+\frac{2}{r+1} f\left(\frac{a+b}{2}\right)-\frac{2}{r(b-a)} \int_{a}^{b} f(x) d x\right|
$$




$$
\leq(b-a)^{2} K(h, r)\left(\left|f^{\prime \prime}(a)\right|+\left|f^{\prime \prime}(b)\right|\right)
$$

where

$$
K(h, r)=\frac{3 r^{2}-9 r+8}{480 r^{2}(r+1)^{2}}+\int_{0}^{1} h^{2}(t) d t .
$$

Proof. From Lemma 1 and properties of absolute value, we have

$$
\begin{aligned}
& \left|\frac{1}{r(r+1)}[f(a)+f(b)]+\frac{2}{r+1} f\left(\frac{a+b}{2}\right)-\frac{2}{r(b-a)} \int_{a}^{b} f(x) d x\right| \\
& \leq(b-a)^{2} \int_{0}^{1}|k(t)|\left|f^{\prime \prime}(t b+(1-t) a)\right| d t \\
& =(b-a)^{2}\left\{\int_{0}^{\frac{1}{2}}\left|\frac{t}{r}\left(\frac{1}{r+1}-t\right)\right|\left|f^{\prime \prime}(t b+(1-t) a)\right| d t\right. \\
& \left.+\int_{\frac{1}{2}}^{1}\left|(1-t)\left(\frac{t}{r}-\frac{1}{r+1}\right)\right|\left|f^{\prime \prime}(t b+(1-t) a)\right| d t\right\} .
\end{aligned}
$$

Since $\left|f^{\prime \prime}\right|$ is $h$-convex, we get

$$
\begin{aligned}
& \left|\frac{1}{r(r+1)}[f(a)+f(b)]+\frac{2}{r+1} f\left(\frac{a+b}{2}\right)-\frac{2}{r(b-a)} \int_{a}^{b} f(x) d x\right| \\
& \leq(b-a)^{2}\left[\int_{0}^{\frac{1}{2}}\left|\frac{t}{r}\left(\frac{1}{r+1}-t\right)\right|\left|h(t) f^{\prime \prime}(b)+h(1-t) f^{\prime \prime}(a)\right| d t\right. \\
& \left.+\int_{\frac{1}{2}}^{1}\left|(1-t)\left(\frac{t}{r}-\frac{1}{r+1}\right)\right|\left|h(t) f^{\prime \prime}(b)+h(1-t) f^{\prime \prime}(a)\right| d t\right] \\
& \leq(b-a)^{2}\left[\int_{0}^{\frac{1}{2}}\left|\frac{t}{r}\left(\frac{1}{r+1}-t\right)\right|\left\{h(t)\left|f^{\prime \prime}(b)\right|+h(1-t)\left|f^{\prime \prime}(a)\right|\right\} d t\right.
\end{aligned}
$$




$$
\begin{aligned}
& \left.+\int_{\frac{1}{2}}^{1}\left|(1-t)\left(\frac{t}{r}-\frac{1}{r+1}\right)\right|\left\{h(t)\left|f^{\prime \prime}(b)\right|+h(1-t)\left|f^{\prime \prime}(a)\right|\right\} d t\right] \\
& =(b-a)^{2}\left[J_{1}+J_{2}\right] .
\end{aligned}
$$

So we can write

$$
\begin{aligned}
J_{1} & =\int_{0}^{\frac{1}{2}}\left|\frac{t}{r}\left(\frac{1}{r+1}-t\right)\right|\left\{h(t)\left|f^{\prime \prime}(b)\right|+h(1-t)\left|f^{\prime \prime}(a)\right|\right\} d t \\
& =\left|f^{\prime \prime}(a)\right| \int_{0}^{\frac{1}{2}}\left|\frac{t}{r}\left(\frac{1}{r+1}-t\right)\right| h(1-t) d t+\left|f^{\prime \prime}(b)\right| \int_{0}^{\frac{1}{2}}\left|\frac{t}{r}\left(\frac{1}{r+1}-t\right)\right| h(t) d t .
\end{aligned}
$$

Since $c d \leq \frac{1}{2}\left(c^{2}+d^{2}\right)$ for $c, d \in \mathbb{R}$ we have

$$
\begin{aligned}
J_{1} & \leq\left|f^{\prime \prime}(a)\right|\left\{\frac{1}{2}\left[\int_{0}^{\frac{1}{2}}\left(\frac{t}{r}\left(\frac{1}{r+1}-t\right)\right)^{2} d t+\int_{0}^{\frac{1}{2}} h^{2}(1-t) d t\right]\right\} \\
+ & \left|f^{\prime \prime}(b)\right|\left\{\frac{1}{2}\left[\int_{0}^{\frac{1}{2}}\left(\frac{t}{r}\left(\frac{1}{r+1}-t\right)\right)^{2} d t+\int_{0}^{\frac{1}{2}} h^{2}(t) d t\right]\right\} \\
& =\left|f^{\prime \prime}(a)\right|\left\{\frac{1}{2}\left[\frac{3 r^{2}-9 r+8}{480 r^{2}(r+1)^{2}}+\int_{\frac{1}{2}}^{1} h^{2}(t) d t\right]\right\} \\
+ & \left|f^{\prime \prime}(b)\right|\left\{\frac{1}{2}\left[\frac{3 r^{2}-9 r+8}{480 r^{2}(r+1)^{2}}+\int_{0}^{\frac{1}{2}} h^{2}(t) d t\right]\right\} .
\end{aligned}
$$

By similar calculation we get

$$
J_{2} \leq\left|f^{\prime \prime}(a)\right|\left\{\frac{1}{2}\left[\frac{3 r^{2}-9 r+8}{480 r^{2}(r+1)^{2}}+\int_{0}^{\frac{1}{2}} h^{2}(t) d t\right]\right\}
$$




$$
+\left|f^{\prime \prime}(b)\right|\left\{\frac{1}{2}\left[\frac{3 r^{2}-9 r+8}{480 r^{2}(r+1)^{2}}+\int_{\frac{1}{2}}^{1} h^{2}(t) d t\right]\right\} \text {. }
$$

If we use the inequalities (3.3) and (3.4) in (3.2) we get the desired result.

Corollary 6. In the inequality (3.1), if we choose $h(t)=1$ and $r=1$, we get

$$
\begin{aligned}
& \left|\frac{f(a)+f(b)}{2}+f\left(\frac{a+b}{2}\right)-\frac{2}{b-a} \int_{a}^{b} f(x) d x\right| \\
& \leq \frac{37.13}{2^{5} .3 .5}(b-a)^{2}\left(\frac{\left|f^{\prime \prime}(a)\right|+\left|f^{\prime \prime}(b)\right|}{2}\right) .
\end{aligned}
$$

Corollary 7. In Theorem 6, if we choose $h(t)=t$ and $r=2$ we have

$$
\begin{aligned}
& \left|\frac{1}{6}\left[f(a)+4 f\left(\frac{a+b}{2}\right)+f(b)\right]-\frac{1}{b-a} \int_{a}^{b} f(x) d x\right| \\
& \leq \frac{11.131}{2^{5} \cdot 3^{3} \cdot 5}(b-a)^{2}\left(\frac{\left|f^{\prime \prime}(a)\right|+\left|f^{\prime \prime}(b)\right|}{2}\right) .
\end{aligned}
$$

Theorem 5. Let $f: I \subset \mathbb{R} \rightarrow \mathbb{R}$ be a twice differentiable mapping on $I^{\circ}$ such that $f^{\prime \prime} \in L_{1}[a, b]$. If $\left|f^{\prime \prime}\right|^{p}$ is $h$-convex on $[a, b]$, where $a, b \in I$ with $a<b, r \geq 1$ and $p>1$, then the following inequality hold

$$
\begin{aligned}
& \left|\frac{1}{r(r+1)}[f(a)+f(b)]+\frac{2}{r+1} f\left(\frac{a+b}{2}\right)-\frac{2}{r(b-a)} \int_{a}^{b} f(x) d x\right| \\
& \leq \frac{(b-a)^{2}}{4 r}\left\{\frac{2^{q+2}+(r-1)^{q+1}(q r+q+r+3)}{2(r+1)^{q+2}(q+1)(q+2)}\right\} \\
& \times\left\{\left[\left|f^{\prime \prime}(b)\right|^{\frac{1}{q}} A(h, t)+\left|f^{\prime \prime}(a)\right|^{p} B(h, t)\right]^{\frac{1}{p}}\right. \\
& \left.\quad+\left[\left|f^{\prime \prime}(b)\right|^{p} B(h, t)+\left|f^{\prime \prime}(a)\right|^{p} A(h, t)\right]^{\frac{1}{p}}\right\}
\end{aligned}
$$

where $A(h, t)=\frac{1}{24}+\int_{0}^{\frac{1}{2}} h^{2}(t) d t$ and $B(h, t)=\frac{1}{24}+\int_{\frac{1}{2}}^{1} h^{2}(t) d t$. 
Proof. By using Lemma 1, triangle inequality and weighted version of Hölder's inequality we have

$$
\begin{aligned}
& \left|\frac{1}{r(r+1)}[f(a)+f(b)]+\frac{2}{r+1} f\left(\frac{a+b}{2}\right)-\frac{2}{r(b-a)} \int_{a}^{b} f(x) d x\right| \\
& \leq(b-a)^{2} \\
& \times\left\{\left(\int_{0}^{\frac{1}{2}}\left|f^{\prime \prime}(t b+(1-t) a)\right|^{p} \frac{t}{r} d t\right)^{\frac{1}{p}}\left(\int_{0}^{\frac{1}{2}}\left|\frac{1}{r+1}-t\right|^{q} \frac{t}{r} d t\right)^{\frac{1}{q}}\right. \\
& \left.+\left(\int_{\frac{1}{2}}^{1}\left|f^{\prime \prime}(t b+(1-t) a)\right|^{p}(1-t) d t\right)^{\frac{1}{p}}\left(\int_{\frac{1}{2}}^{1}\left|\frac{t}{r}-\frac{1}{r+1}\right|^{q}(1-t) d t\right)^{\frac{1}{q}}\right\}
\end{aligned}
$$

By using $h-$ convexity of $\left|f^{\prime \prime}\right|^{p}$ we have

$$
\begin{aligned}
& \left|\frac{1}{r(r+1)}[f(a)+f(b)]+\frac{2}{r+1} f\left(\frac{a+b}{2}\right)-\frac{2}{r(b-a)} \int_{a}^{b} f(x) d x\right| \\
& \leq(b-a)^{2} \\
& \times\left\{\left(\int_{0}^{\frac{1}{2}}\left[h(t)\left|f^{\prime \prime}(b)\right|^{p}+h(1-t)\left|f^{\prime \prime}(a)\right|^{p}\right] \frac{t}{r} d t\right)^{\frac{1}{p}}\left(\int_{0}^{\frac{1}{2}}\left|\frac{1}{r+1}-t\right|^{q} \frac{t}{r} d t\right)^{\frac{1}{q}}\right. \\
& +\left(\int_{\frac{1}{2}}^{1}\left[h(t)\left|f^{\prime \prime}(b)\right|^{p}+h(1-t)\left|f^{\prime \prime}(a)\right|^{p}\right](1-t) d t\right)^{\frac{1}{p}} \\
& \left.\quad \int_{\frac{1}{2}}^{1}\left|\frac{t}{r}-\frac{1}{r+1}\right|^{q}(1-t) d t\right)^{\frac{1}{q}} \\
& \quad=(b-a)^{2}
\end{aligned}
$$




$$
\begin{aligned}
& \times\left\{\left(\frac{\left|f^{\prime \prime}(b)\right|^{p}}{r} \int_{0}^{\frac{1}{2}} t h(t) d t+\frac{\left|f^{\prime \prime}(a)\right|^{p}}{r} \int_{0}^{\frac{1}{2}} t h(1-t) d t\right)^{\frac{1}{p}}\right. \\
& +\left(\int_{0}^{\frac{1}{2}}\left|\frac{1}{r+1}-t\right|^{q} t d t\right)^{\frac{1}{q}} \\
& +\left(\left|f^{\prime \prime}(b)\right|^{p} \int_{\frac{1}{2}}^{1}(1-t) h(t) d t+\left|f^{\prime \prime}(a)\right|^{p} \int_{\frac{1}{2}}^{1}(1-t) h(1-t) d t\right)^{\frac{1}{p}} \\
& \\
& \qquad \int_{\left.\frac{1}{2}\left|\frac{t}{r}-\frac{1}{r+1}\right|^{q}(1-t) d t\right)^{\frac{1}{q}}}^{1} .
\end{aligned}
$$

Since $c d \leq \frac{1}{2}\left(c^{2}+d^{2}\right)$ for $c, d \in \mathbb{R}$ we have

$$
\begin{aligned}
& \left|\frac{1}{r(r+1)}[f(a)+f(b)]+\frac{2}{r+1} f\left(\frac{a+b}{2}\right)-\frac{2}{r(b-a)} \int_{a}^{b} f(x) d x\right| \\
& \leq(b-a)^{2}\left\{\left(\int_{0}^{\frac{1}{2}}\left|\frac{1}{r+1}-t\right|^{q} t d t\right)^{\frac{1}{q}}\right. \\
& \quad \times\left[\frac{\left|f^{\prime \prime}(b)\right|^{p}}{2 r}\left(\int_{0}^{\frac{1}{2}} t^{2} d t+\int_{0}^{\frac{1}{2}} h^{2}(t) d t\right)\right. \\
& \left.+\frac{\left|f^{\prime \prime}(a)\right|^{p}}{2 r}\left(\int_{0}^{\frac{1}{2}} t^{2} d t+\int_{0}^{\frac{1}{2}} h^{2}(1-t) d t\right)\right]^{\frac{1}{p}}
\end{aligned}
$$




$$
\begin{gathered}
+\left(\int_{\frac{1}{2}}^{1}\left|\frac{t}{r}-\frac{1}{r+1}\right|^{q}(1-t) d t\right)^{\frac{1}{q}} \times\left[\frac{\left|f^{\prime \prime}(b)\right|^{p}}{2}\left(\int_{\frac{1}{2}}^{1}(1-t)^{2} d t+\int_{\frac{1}{2}}^{1} h^{2}(t) d t\right)\right. \\
\left.+\frac{\left|f^{\prime \prime}(a)\right|^{p}}{2}\left(\int_{\frac{1}{2}}^{1}(1-t)^{2} d t+\int_{\frac{1}{2}}^{1} h^{2}(1-t) d t\right)\right]^{\frac{1}{p}}
\end{gathered}
$$

If we simplify the expression, we have

$$
\begin{aligned}
& \left|\frac{1}{r(r+1)}[f(a)+f(b)]+\frac{2}{r+1} f\left(\frac{a+b}{2}\right)-\frac{2}{r(b-a)} \int_{a}^{b} f(x) d x\right| \\
& \leq \frac{(b-a)^{2}}{4 r}\left\{\frac{2^{q+2}+(r-1)^{q+1}(q r+q+r+3)}{2(r+1)^{q+2}(q+1)(q+2)}\right\}^{\frac{1}{q}} \\
& \times\left\{\left.|| f^{\prime \prime}(b)\right|^{p}\left(\frac{1}{24}+\int_{0}^{\frac{1}{2}} h^{2}(t) d t\right)+\left|f^{\prime \prime}(a)\right|^{p}\left(\frac{1}{24}+\int_{\frac{1}{2}}^{1} h^{2}(t) d t\right)\right]^{\frac{1}{p}} \\
& \left.+\left[\left|f^{\prime \prime}(b)\right|^{p}\left(\frac{1}{24}+\int_{\frac{1}{2}}^{1} h^{2}(t) d t\right)+\left|f^{\prime \prime}(a)\right|^{p}\left(\frac{1}{24}+\int_{0}^{\frac{1}{2}} h^{2}(t) d t\right)\right]^{\frac{1}{p}}\right\}
\end{aligned}
$$

then the proof is complete.

Corollary 8. If we choose $h(t)=1, r=1$ in the inequality (3.5) we get

$$
\begin{aligned}
& \left|\frac{f(a)+f(b)}{2}+f\left(\frac{a+b}{2}\right)-\frac{2}{b-a} \int_{a}^{b} f(x) d x\right| \\
& \leq \frac{(b-a)^{2}}{4}\left\{\frac{1}{(q+1)(q+2)}\right\}^{\frac{1}{q}}\left\{\frac{13}{12}\left(\left|f^{\prime \prime}(a)\right|^{p}+\left|f^{\prime \prime}(b)\right|^{p}\right)\right\}^{\frac{1}{p}} .
\end{aligned}
$$

Corollary 9. If we choose $h(t)=1, r=2$ in (3.5), we get

$$
\left|\frac{1}{6}\left[f(a)+4 f\left(\frac{a+b}{2}\right)+f(b)\right]-\frac{1}{b-a} \int_{a}^{b} f(x) d x\right|
$$




$$
\leq \frac{(b-a)^{2}}{4}\left\{\frac{2^{q+2}+(3 q+5)}{2.3^{q+2}(q+1)(q+2)}\right\}^{\frac{1}{q}}\left\{\frac{13}{24}\left(\left|f^{\prime \prime}(a)\right|^{p}+\left|f^{\prime \prime}(b)\right|^{p}\right)\right\}^{\frac{1}{p}} .
$$

Corollary 10. If we choose $h(t)=t, r=2$ in the inequality (3.5), we have

$$
\begin{aligned}
& \left|\frac{1}{6}\left[f(a)+4 f\left(\frac{a+b}{2}\right)+f(b)\right]-\frac{1}{b-a} \int_{a}^{b} f(x) d x\right| \\
& \leq \frac{(b-a)^{2}}{8}\left\{\frac{2^{q+2}+(3 q+5)}{2.3^{q+2}(q+1)(q+2)}\right\}^{\frac{1}{q}} \\
& \times\left\{\left(\frac{\left|f^{\prime \prime}(a)\right|^{p}}{12}+\frac{\left|f^{\prime \prime}(b)\right|^{p}}{3}\right)^{\frac{1}{p}}+\left(\frac{\left|f^{\prime \prime}(a)\right|^{p}}{3}+\frac{\left|f^{\prime \prime}(b)\right|^{p}}{12}\right)^{\frac{1}{p}}\right\} .
\end{aligned}
$$

Corollary 11. In addition to the conditions given above, if we choose $p=q=2$, we have

$$
\begin{aligned}
& \left|\frac{1}{6}\left[f(a)+4 f\left(\frac{a+b}{2}\right)+f(b)\right]-\frac{1}{b-a} \int_{a}^{b} f(x) d x\right| \\
& \leq \frac{(b-a)^{2}}{48 \sqrt{2}}\left\{\left(\frac{\left|f^{\prime \prime}(a)\right|^{2}}{12}+\frac{\left|f^{\prime \prime}(b)\right|^{2}}{3}\right)^{\frac{1}{2}}+\left(\frac{\left|f^{\prime \prime}(a)\right|^{2}}{3}+\frac{\left|f^{\prime \prime}(b)\right|^{2}}{12}\right)^{\frac{1}{2}}\right\} .
\end{aligned}
$$

\section{REFERENCES}

[1] M. Alomari, M. Darus, and S. S. Dragomir, "New inequalities of Simpson's type for s-convex functions with applications," RGMIA Res. Rep. Coll., vol. 12, no. 4, Article 9, 2009.

[2] S. S. Dragomir, "Two mappings in connection to Hadamard's inequalities," J. Math. Anal. Appl., vol. 167, no. 1, pp. 49-56, 1992.

[3] S. Dragomir, R. Agarwal, and P. Cerone, "On Simpson's inequality and applications," J. Inequal. Appl., vol. 5, no. 6, pp. 533-579, 2000.

[4] U. gur S. Kirmaci, "Inequalities for differentiable mappings and applications to special means of real numbers and to midpoint formula," Appl. Math. Comput., vol. 147, no. 1, pp. 137-146, 2004.

[5] Z. Liu, “An inequality of Simpson type," Proc. R. Soc. Lond., Ser. A, Math. Phys. Eng. Sci., vol. 461, no. 2059, pp. 2155-2158, 2005.

[6] M. E. Özdemir, A. Ekinci, and A. O. Akdemir, "Generalizations of integral inequalities for functions whose second derivatives are convex and $m$-convex," Miskolc Math. Notes, vol. 13, no. 2, pp. 441-457, 2012.

[7] J. E. Pečarić, F. Proschan, and Y. Tong, Convex functions, partial orderings, and statistical applications, ser. Mathematics in Science and Engineering. Boston, MA: Academic Press, 1992, vol. 187.

[8] M. Sarıkaya, E. Set, and M. Özdemir, "On new inequalities of simpson's type for functions whose second derivatives absolute values are convex," RGMIA Res. Rep. Coll., vol. 13, no. 1, Article 1, 2010. 
[9] G. Toader, "On a generalization of the convexity," Math., Rev. Anal. Numér. Théor. Approximation, Math., vol. 30, no. 1, pp. 83-87, 1988.

[10] S. Varošanec, "On h-convexity," J. Math. Anal. Appl., vol. 326, no. 1, pp. 303-311, 2007.

[11] G.-S. Yang, D.-Y. Hwang, and K.-L. Tseng, "Some inequalities for differentiable convex and concave mappings," Comput. Math. Appl., vol. 47, no. 2-3, pp. 207-216, 2004.

Authors' addresses

\section{Emin Özdemir}

Atatürk University, K. K. Education Faculty, Department of Mathematics, 25240, Campus, Erzurum, Turkey

E-mail address: emoseatauni.edu.tr

\section{Mustafa Gürbüz}

Graduate School of Natural and Applied Sciences, Ağri İbrahim Çeçen University, Ağri, Turkey

E-mail address: mgurbuzeagri.edu.tr

\section{Çetin Yildiz}

Atatürk University, K. K. Education Faculty, Department of Mathematics, 25240, Campus, Erzurum, Turkey

E-mail address: cetin@atauni.edu.tr 